TITLE:

\title{
Manipulation of mica flakes using photothermally generated microbubbles
}

$\operatorname{AUTHOR}(S):$

Namura, Kyoko; Matsumura, Ryuta; Kumar, Samir; Suzuki, Motofumi

\section{CITATION:}

Namura, Kyoko ... [et al]. Manipulation of mica flakes using photothermally generated microbubbles. Proceedings of SPIE - The International Society for Optical Engineering 2020, 11522: 115220H.

ISSUE DATE:

2020-06-15

URL:

http://hdl.handle.net/2433/253770

\section{RIGHT:}

Copyright 2020 Society of Photo-Optical Instrumentation Engineers (SPIE). One print or electronic copy may be made for personal use only. Systematic reproduction and distribution, duplication of any material in this publication for a fee or for commercial purposes, and modification of the contents of the publication are prohibited. 


\section{Manipulation of mica flakes using photothermally generated microbubbles}

Namura, Kyoko, Matsumura, Ryuta, Kumar, Samir, Suzuki, Motofumi

Kyoko Namura, Ryuta Matsumura, Samir Kumar, Motofumi Suzuki, "Manipulation of mica flakes using photothermally generated microbubbles," Proc. SPIE 11522, Optical Manipulation and Structured Materials Conference 2020, 115220H (15 June 2020); doi: 10.1117/12.2573517

EDIE Event: SPIE Technologies and Applications of Structured Light, 2020, Yokohama, Japan 


\title{
Manipulation of mica flakes using photothermally generated microbubbles*
}

\author{
Kyoko Namura*a, Ryuta Matsumura ${ }^{\text {a }}$,Samir Kumara, Motofumi Suzuki ${ }^{\mathrm{a}}$ \\ aDepartment of Micro Engineering, Kyoto University, Kyoto Daigaku-Katsura, Nishikyo-ku, Kyoto, \\ 615-8540, Japan
}

\begin{abstract}
We experimentally investigated the manipulation of mica flakes using photothermally induced microbubbles. Iron silicide was sputtered on mica flakes to absorb the laser light and convert it to heat. By focusing a laser on to the flake immersed in degassed water, a water vapor microbubble was generated on the flake. The bubble involved strong Marangoni flow due to the steep temperature gradient on it. Laser irradiation at multiple spots allowed us to control the direction of the temperature gradient and subsequent Marangoni flow. By generating the flow parallel to the flake surface, the flake was driven on the glass substrate. This method is expected as a novel method to manipulate large and heavy particles in microfluidic channels.
\end{abstract}

Keywords: microbubble, photothermal conversion, mica flakes, spatial light modulator, Marangoni flow, degassing

\section{Introduction}

Manipulation of microscale objects in microfluidic channels has been attracted much attention upon the development of lab-on-a-chip devices ${ }^{1}$. The techniques are expected to be useful for such as manipulating biological materials ${ }^{2}$ and control microfluidic flows ${ }^{3}$. The most widely used technique is, of course, the optical tweezers ${ }^{4}$. This method gives extremely flexible ways to manage microscale objects in various liquids. However, the typical force is on the order of $\mathrm{pN}$ under the realistic laser power of several $\mathrm{W}$, and the method is sometimes not sufficient to drive micro-objects rapidly.

Recently, we have reported a rapid flow generation around a water vapor microbubble in degassed water using the thermoplasmonic effect of a gold nanoisland film ${ }^{5,6}$. By focusing a laser on the gold nanoisland film immersed in degassed water, a water vapor microbubble with a diameter of $\sim 10 \mu \mathrm{m}$ was generated on the laser spot. Under continuous heating, the water vapor bubble involved significantly rapid flows compared to an air bubble generated in non-degassed water. This flow generation is mainly because of the Marangoni effect, which is induced by the surface tension imbalance under a temperature gradient. The flow field around the water vapor bubble was well described using a point force, i.e., a stokeslet. The strength of the stokeslet was estimated to

*namura@me.kyoto-u.ac.jp; phone 81753833697 be $0.01-0.1 \mu \mathrm{N}$ for the laser power of $40-50 \mathrm{~mW}$. Besides, the direction of the stokeslet was tunable by irradiating laser at multiple spots. First, a primary laser spot focused on the gold nanoisland film can generate a water vapor bubble. By adding a sub laser spot next to the primary laser spot, a temperature gradient in the direction parallel to the substrate surface is applied to the bubble. Finally, the flow direction around the bubble tilted from the direction normal to the substrate surface, and rapid flow in the direction parallel to the substrate surface was generated. This technique allowed us to apply a force on the microfluid in the direction parallel to the wall surface. In our previous study, rapid flow is generated because the substrate was fixed. If the substrate is mobile, the bubble may drive the substrate instead of generating rapid flow.

In this study, we focused our attention on a mica flake. The mica flake coated with iron silicide will be used as a mobile substrate with a photothermal conversion property. We demonstrate the manipulation of mica flake using the photothermally generated microbubble in degassed water.

\section{Experiments}

We prepared $\mathrm{FeSi}_{2}$ thin film of $50 \mathrm{~nm}$ on a mica substrate using RF magnetron sputtering technique. After the thin film preparation, the mica was cleaved as thin as possible and cut into small pieces of less than $1 \mathrm{~mm}$ square. The degassed water was prepared by sonicating ultrapure water (18.2 $\mathrm{M} \Omega \mathrm{cm}$ from Millipore-Direct Q 
UV3, Merck) under vacuum $\left(\sim 3 \mathrm{kPa}\right.$ at $\left.25^{\circ} \mathrm{C}\right)$. Then, the mica flakes and degassed water was sealed in a fluidic cell.

In order to perform the manipulation of the mica flakes, the fluidic cell was placed in our observation system, which includes an upright microscope for observation and a laser irradiation system for thermoplasmonic heating (Fig. 1). The upright microscope used for the observation of the fluidic phenomena in the cell was equipped with an objective lens $(10 \times, \mathrm{NA}=0.26)$. The observed region was at the bottom of the fluidic cell, where mica flakes were found. A short-pass filter was placed in front of the camera to eliminate the $835-\mathrm{nm}$ laser source that was used for the photothermal heating.

The laser irradiation at multiple spots on the mica flakes was performed using a spatial light modulator (SLM). The detailed method is described in our previous paper $^{6}$. A primary laser spot for the bubble formation was irradiated on $\mathrm{FeSi}_{2}$ film, which had a fixed power of $P_{\text {primary }}=36 \pm 2 \mathrm{~mW}$. Subsequently, a sub laser spot was added next to the primary spot to provide an additional temperature gradient on the bubble surface. The power of the sub laser spot, $P_{s u b}$, was varied from 0 to $32 \mathrm{~mW}$. The distance between primary and sub laser spots was fixed to $5 \mu \mathrm{m}$. The FWHM of each laser spot was $\sim 2 \mu \mathrm{m}$.

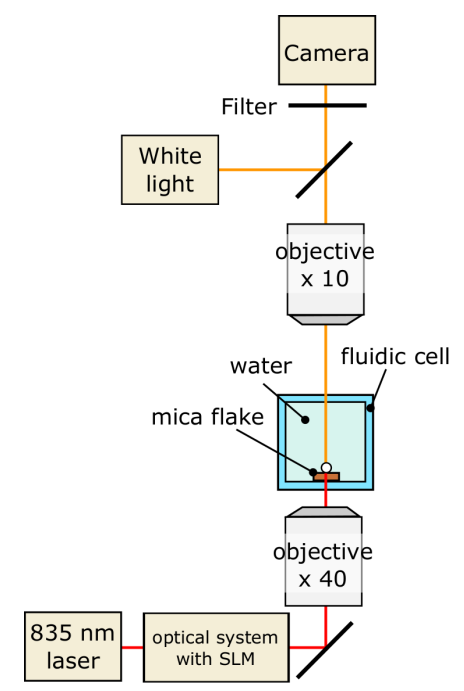

Figure 1. Schematic drawings of the experimental setup.

\section{Results and Discussion}

In order to evaluate the bubble and flow generation due to the photothermal effect of the $\mathrm{FeSi}_{2}$ films on mica, we first used large and fixed mica substrate coated with the $\mathrm{FeSi}_{2}$ film before using flakes. The behavior of the bubble and subsequent flow generation was essentially the same as what observed on the glass substrate coated with the gold nanoisland film ${ }^{3}$. The rapid flow in the direction perpendicular to the substrate surface was observed at $P_{s u b}$ $=0 \mathrm{~mW}$, whereas the rapid flow in the direction parallel to the substrate was observed at $P_{s u b}=15 \mathrm{~mW}$.

Then, the bubble was generated on mica flakes. When the flake is irradiated with only the primally laser spot, it did not move. On the other hand, when $P_{s u b}=15 \mathrm{~mW}$, the flake was driven in the direction parallel to the mica surface. In most cases, the flake moved to the direct primary to sub laser spot. The typical speed of the mica flake was several $\mathrm{mm} / \mathrm{s}$. Instead of generating flow, the tiny mica flake was driven by forces induced by the bubble.

\section{Conclusion}

In this study, we investigated the manipulation of the mica flakes using photothermally induced microbubble. By focusing the laser onto the mica coated with $\mathrm{FeSi}_{2}$ film immersed in degassed water, a water vapor microbubble was generated on it. Under the steep temperature gradient, the bubble generated force and apply it to mica. When the bubble felt an effective temperature gradient in the direction parallel to the mica surface, it drove mica flake in that direction at a speed of several $\mathrm{mm} / \mathrm{s}$. We have successfully demonstrated that the water vapor bubble under multiple laser irradiation is a great candidate for the manipulation of a micro object $\mathrm{n}$ microfluidic channel.

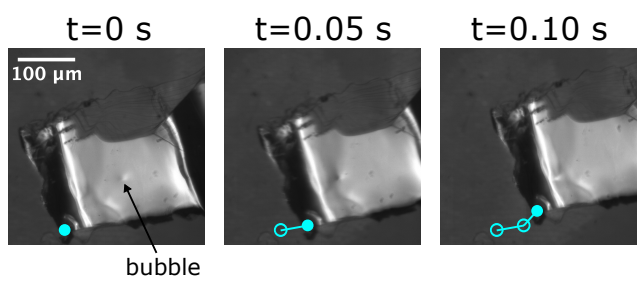

Figure 2. Mica flake motion captured at $\mathrm{t}=0,0.05$, and $0.10 \mathrm{~s}$ under laser irradiation with $P_{s u b}=15 \mathrm{~mW}$. 


\section{References}

[1] Figeys, D. and Pinto, D., "Lab-on-a-chip: A revolution in biological and medical sciences," Anal. Chem. 72, 330A-335A (2000).

[2] Wang, M. et al., "Microfluidic sorting of mammalian cells by optical force switching," Nat. Biotechnol. 23(1), 83-87 (2005).

[3] Baigl, D. "Photo-actuation of liquids for light-driven microfluidics: state of the art and perspectives," Lab Chip 12(19), 3637-3653 (2012).

[4] Ashkin, A., Dziedzic, J. M., and Yamane, T., "Optical trapping and manipulation of single cells using infrared laser beams," Nature 330(6150), 769-771 (1987).

[5] Namura, K., Nakajima, K., and Suzuki, M., "Quasistokeslet induced by thermoplasmonic Marangoni effect around a water vapor microbubble," Sci. Rep. 7, 45776 (2017).

[6] Namura, K., Imafuku, S., Kumar, S., Nakajima, K., Sakakura, M., and Suzuki, M., " Direction control of quasi-stokeslet induced by thermoplasmonic heating of a water vapor microbubble," Sci. Rep. 9(1), 4770 (2019). 